\title{
Noninvasive hematocrit assessment for cardiovascular magnetic resonance extracellular volume quantification using a point-of-care device and synthetic derivation
}

Sean Robison ${ }^{1}$, Gauri Rani Karur ${ }^{1}$, Rachel M. Wald ${ }^{1,2}$, Paaladinesh Thavendiranathan ${ }^{1,2}$, Andrew M. Crean ${ }^{1,2}$ and Kate Hanneman ${ }^{{ }^{*}}$

\begin{abstract}
Background: Calculation of cardiovascular magnetic resonance (CMR) extracellular volume (ECV) requires input of hematocrit, which may not be readily available. The purpose of this study was to evaluate the diagnostic accuracy of ECV calculated using various noninvasive measures of hematocrit compared to ECV calculated with input of laboratory hematocrit as the reference standard.

Methods: One hundred twenty three subjects (47.7 \pm 14.1 years; $42 \%$ male) were prospectively recruited for CMR T1 mapping between August 2016 and April 2017. Laboratory hematocrit was assessed by venipuncture. Noninvasive hematocrit was assessed with a point-of-care (POC) device (Pronto-7 ${ }^{\circ}$ Pulse CO-Oximeter , Masimo Personal Health, Irvine, California, USA) and by synthetic derivation based on the relationship with blood pool T1 values. Left ventricular ECV was calculated with input of laboratory hematocrit (Lab-ECV), POC hematocrit (POC-ECV), and synthetic hematocrit (synthetic-ECV), respectively. Statistical analysis included Wilcoxon signed-rank test, Bland-Altman analysis, receiveroperating curve analysis and intra-class correlation (ICC).

Results: There was no significant difference between Lab-ECV and POC-ECV ( $27.1 \pm 4.7 \%$ vs. $27.3 \pm 4.8 \%, p=0.106)$, with minimal bias and modest precision (bias $-0.18 \%, 95 \% \mathrm{Cl}[-2.85,2.49])$. There was no significant difference between Lab-ECV and synthetic-ECV $(26.7 \pm 4.4 \%$ vs. $26.5 \pm 4.3 \%, p=0.084)$ in subjects imaged at $1.5 \mathrm{~T}$, although bias was slightly higher and limits of agreement were wider (bias $0.23 \%, 95 \% \mathrm{Cl}[-2.82,3.27]$ ). For discrimination of abnormal Lab-ECV $\geq 30 \%$, POC-ECV had good diagnostic performance (sensitivity $85 \%$, specificity $96 \%$, accuracy $94 \%$, and AUC 0.902) and synthetic-ECV had moderate diagnostic performance (sensitivity $71 \%$, specificity $98 \%$, accuracy $93 \%$, and AUC 0.849). POCECV had excellent test-retest (ICC 0.994, 95\%CI[0.987, 0.997]) and inter-observer agreement (ICC 0.974, 95\%CI[0.929, 0.991]).

Conclusions: Myocardial ECV can be accurately and reproducibly calculated with input of hematocrit measured using a noninvasive POC device, potentially overcoming an important barrier to implementation of ECV. Further evaluation of synthetic ECV is required prior to clinical implementation.
\end{abstract}

Keywords: Cardiovascular magnetic resonance (CMR), T1 mapping, Extracellular volume (ECV), Noninvasive hemoglobin monitoring, Hematocrit

\footnotetext{
* Correspondence: kate.hanneman@uhn.ca

'Department of Medical Imaging, Toronto General Hospital, University Health

Network, University of Toronto, 585 University Ave, 1PMB-298, Toronto, ON

M5G 2N2, Canada

Full list of author information is available at the end of the article
}

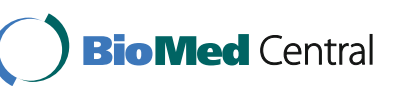

(c) The Author(s). 2018 Open Access This article is distributed under the terms of the Creative Commons Attribution 4.0 International License (http://creativecommons.org/licenses/by/4.0/), which permits unrestricted use, distribution, and reproduction in any medium, provided you give appropriate credit to the original author(s) and the source, provide a link to the Creative Commons license, and indicate if changes were made. The Creative Commons Public Domain Dedication waiver (http://creativecommons.org/publicdomain/zero/1.0/) applies to the data made available in this article, unless otherwise stated. 


\section{Background}

Myocardial extracellular volume (ECV) fraction derived from cardiovascular magnetic resonance (CMR) T1 mapping has been validated based on histology [1-3], with demonstrated prognostic significance in several conditions [4-6]. Calculation of ECV requires accurate and timely assessment of hematocrit, to correct for the blood contrast volume of distribution [7]. Traditional laboratory determination of hematocrit via venipuncture in the setting of ECV evaluation is costly, mildly uncomfortable for the patient, and often inconvenient. Therefore, rapid noninvasive determination of hematocrit would be beneficial in the setting of ECV analysis.

Noninvasive point-of-care (POC) devices have recently become commercially available, providing immediate hemoglobin and hematocrit results without the need for blood sampling by venipuncture or finger prick [8-10]. Additionally, synthetic derivation of hematocrit has recently been described based on the relationship between hematocrit and non-contrast blood pool T1 values [11]. However, there are important limitations to synthetic analysis, as each specific acquisition scheme requires derivation and validation [12]. A recent study suggests that synthetic ECV may result in miscategorization of individual patients [13].

The purpose of this study was to evaluate the diagnostic accuracy of CMR ECV calculated using noninvasive measures of hematocrit (determined with a POC device and synthetic derivation) compared to ECV calculated with input of laboratory hematocrit as the reference standard. We hypothesized that there would be no significant difference in myocardial ECV calculated using noninvasive measures of hematocrit as compared with ECV derived from laboratory hematocrit.

\section{Methods \\ Study population}

Institutional research ethics board approval was obtained for this prospective study. Between August 2016 and April 2017, 149 subjects undergoing clinical or research CMR were prospectively recruited for T1 mapping with calculation of ECV. Written informed consent was obtained from all study subjects. Exclusion criteria included incomplete or aborted CMR $(n=4)$, subject refusal of intravenous contrast administration $(n=2)$, substantial artifact precluding image analysis $(n=4)$, and failure of POC device analysis (due to the presence of gel nail polish, marked hand tremor, and/or inadequate signal) $(n=16)$. The final cohort of 123 subjects included both patients and healthy subjects $(47.7 \pm 14.1$ years; $42 \%$ male). Clinical and demographic information were extracted from the electronic patient record.

\section{CMR technique}

CMR was performed at $1.5 \mathrm{~T}(n=74$, Magnetom Avanto, Siemens Healthineers, Erlangen, Germany) or 3 T $(n=34$, Magnetom Skyra, Siemens Healthineers; and $n=15$ Biograph mMR, Siemens Healthineers). Retrospectively gated balanced steady-state free precession (bSSFP) cine images were obtained for assessment of left ventricular (LV) size and function by a stack of short-axis slices with coverage from cardiac base to the apex with $8-\mathrm{mm}$ thickness and $2-\mathrm{mm}$ inter-slice gap. T1 mapping was performed using a bSSFP readout modified Look-Locker inversion recovery (MOLLI) acquisition scheme with three short-axis slices (8 $\mathrm{mm}$ slice thickness) acquired at basal, mid and apical locations, pre-contrast and 12-15 min after administration of $0.15 \mathrm{mmol} / \mathrm{kg}$ body weight of gadobuterol (Gadovist; Bayer Healthcare, Berlin, Germany) [14]. Multi-plane late gadolinium enhanced (LGE) imaging was performed approximately $15 \mathrm{~min}$ following contrast administration employing a $2 \mathrm{D}$ inversion recovery gradient-recalled echo sequence (slice thickness $8 \mathrm{~mm}$ and $2 \mathrm{~mm}$ inter-slice gap).

\section{CMR analysis}

All studies were analyzed by a fellowship trained radiologist (SR, with 3 years CMR experience) blinded to all identifying clinical and imaging data. Evaluation of LV volumes and function was performed using commercially available software (QMASS MRI, Medis Medical Imaging Systems, Leiden, The Netherlands).

After inline, non-rigid motion correction of individual T1 mapping images, an inline T1 map was generated using standard 3-parameter fitting. Analysis was performed off-line using commercially available software (cvi42; Circle Cardiovascular Imaging, Calgary, Alberta, Canada). Regions of interest were drawn manually in the LV blood pool on pre- and post-contrast T1 maps with care taken to avoid the myocardium and papillary muscles. Myocardial T1 values were calculated by contouring epicardial and endocardial borders at basal, mid and apical slices, with care taken to avoid blood pool and epicardial fat, Fig. 1. Myocardial segments with LGE were not excluded from T1 analysis. Blood pool and myocardial $\mathrm{T} 1$ values were averaged across the three slices.

\section{Hematocrit assessment}

A small blood sample $(<30 \mathrm{~mL})$ was collected by venipuncture within $24 \mathrm{~h}$ of CMR for the purpose of central hospital laboratory determination of hemoglobin and hematocrit (Lab-Hct). The time between laboratory blood sampling and CMR start time was recorded. Samples were analyzed by a hematology analyzer (CELLDYN, Sapphire, Abbott Core Laboratory, Abbott Park, 


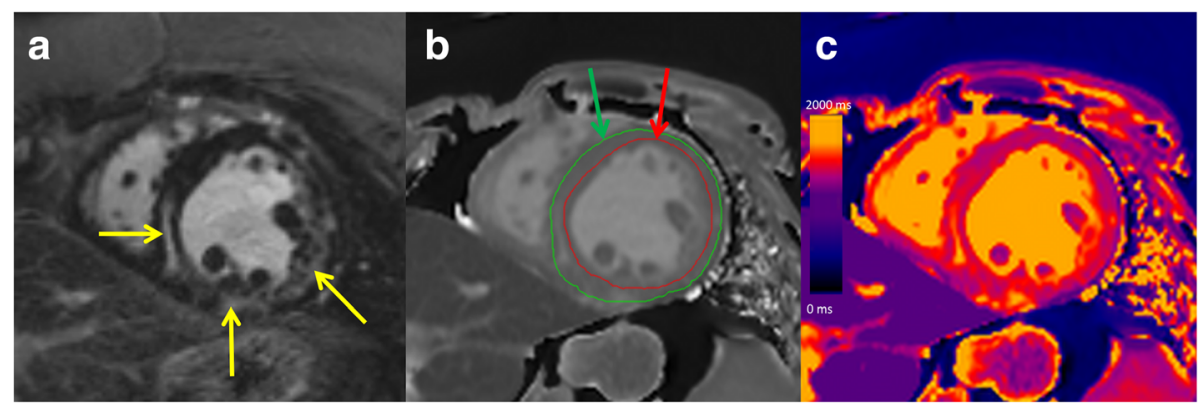

Fig. 1 Example short axis late gadolinium enhanced (LGE) image (a), non-contrast T1 map with contours (b), and color non-contrast T1 map (c) in a 30-year-old female with cardiac sarcoidosis. Yellow arrows indicate the presence of LGE. Endocardial (red arrow) and epicardial (green arrow) contours are shown

Illinois, USA), with reported bias and standard deviation of $-0.07 \pm 1.17 \mathrm{~g} / \mathrm{dL}[15]$. Laboratory hemoglobin and hematocrit results were recorded blinded to clinical information and the results of POC and synthetic derivation analysis. Anemia was defined as a laboratory hemoglobin level $<122 \mathrm{~g} / \mathrm{L}$ for females, $<132 \mathrm{~g} / \mathrm{L}$ for males $\geq 60$ years of age, and $<137 \mathrm{~g} / \mathrm{L}$ for males $<60$ years of age [16].

POC analysis was performed within $1 \mathrm{~h}$ of CMR using a Pronto- $7^{\circ}$ Pulse CO-Oximeter (Masimo Personal Health, Irvine, California, USA), by an experienced observer (SR) blinded to all identifying information and the results of laboratory analysis and synthetic derivation. The POC probe was applied to the fingertip of the ring or middle finger according to the manufacturer's guidelines. If the first attempt was unsuccessful with no result displayed, up to four additional attempts at POC analysis were performed. The total number of POC analysis attempts was recorded for each subject. The displayed hemoglobin value was recorded with subsequent derivation of hematocrit (POC-Hct) using a conversion of 0.3, according to previously published guidelines [17]. In a subset of subjects $(n=32)$, the total time for POC device analysis was determined, timed from turning the device on to recording the displayed hemoglobin value.

In the subgroup of subjects scanned at $1.5 \mathrm{~T}$, synthetic hematocrit (synthetic-Hct) was calculated from the relationship between hematocrit and non-contrast blood pool T1 values as described previously, using a published linear regression formula for MOLLI acquisition schemes at $1.5 \mathrm{~T}$ [11]:

$$
\text { Synthetic-Hct }=\left(866.0 *\left[1 / \mathrm{T}_{\text {blood }}\right]\right)-0.1232
$$

Synthetic hematocrit was calculated by an experienced observer (SR) who was blinded to clinical information and the results of POC and laboratory analysis.
Synthetic-hematocrit was not evaluated in subjects imaged at $3 \mathrm{~T}$ due to lack of previously published formulas using the same CMR scanner that was used in this study. We did not fit a linear regression equation to our data given the relatively small number of subjects imaged at each field strength and acquisition scheme.

\section{ECV derivation}

Myocardial ECV was calculated based on pre- and postcontrast myocardial and blood pool $\mathrm{T} 1$ values and hematocrit, as proposed by Arheden et al. [18]. ECV was calculated with input of Lab-Hct (Lab-ECV), POC-Hct (POC-ECV), and synthetic-Hct (synthetic-ECV), respectively. The same myocardial and blood pool T1 values were used in each ECV calculation.

\section{Test-retest and inter-observer agreement}

To evaluate test-retest variability of POC measures, three POC analyses were performed in immediate succession in a subset of subjects $(n=42)$ without removal of the probe. In these subjects, the first result obtained was used in the general analysis.

To assess inter-observer agreement of POC measures, a second POC analysis was performed in a subset of subjects $(n=16)$ by a second experienced CMR fellowship trained reader (GK) independent from the first analysis, blinded to all identifying clinical data and the first set of measurements.

\section{Comparison of POC devices}

Evaluation of hematocrit using a second POC device (Pronto Pulse CO-Oximeter, Masimo Personal Health) was performed in a group of 10 subjects. These 10 subjects also underwent parallel assessment using the Pronto- $7^{\circ}$ POC device and central hospital laboratory determination. Myocardial ECV was calculated in this group with input of hematocrit from the Pronto device, Pronto- $7^{\circ}$ device, and laboratory determination. 


\section{Statistical analysis}

Statistical analysis was performed using STATA v14.1 (Stata Corporation, College Station, Texas, USA). The sample size was calculated to detect a difference in ECV measurements between techniques of $1 \%$ with a standard deviation of ECV measurements of 3\% using a paired t-test [19]. To detect this difference with a power of $95 \%$ and alpha error of 0.05 , a total of 119 subjects were needed. All continuous data were first tested for normal distribution using the Shapiro-Wilk test. Continuous variables are described using mean and standard deviation or median and interquartile range (IQR), and categorical variables using numbers and percentage. Comparisons between values were made by paired $t$-test for continuous values with normal distribution and Wilcoxon signed-rank test for continuous values with nonnormal distribution. Correlations between continuous variables were assessed with Pearson or Spearman correlation coefficient, as appropriate. Bias and precision were evaluated using Bland-Altman analysis. Test-retest and inter-observer agreement were assessed via the intra-class correlation coefficient (ICC) with two-way random effects model. Sensitivity analysis was performed, restricting the analysis to subjects in different subgroups including those with anemia, different field strengths (1.5 $\mathrm{T}$ and $3 \mathrm{~T}$ ), gender (males and females) and clinical status (healthy subjects and patients). Diagnostic test performance of POC-ECV and synthetic-ECV in comparison to Lab-ECV as the reference standard was assessed using a Lab-ECV cut-off value of $\geq 30 \%$ as abnormal, including sensitivity, specificity, accuracy and area under the receiver operating curve (ROC) $[20,21]$. A two-tailed $p$-value of $<0.05$ was considered statistically significant.

\section{Results}

Baseline characteristics and clinical details are summarized in Table 1. CMR findings are summarized in Table 2.

\section{Hematocrit assessment and ECV derivation}

Hemoglobin, hematocrit and ECV values are detailed in Table 2. The median interval between blood sampling and CMR was $1.3 \mathrm{~h}$ (IQR 1.0-2.8 h). Mean laboratory hemoglobin and Lab-Hct values were $134.1 \pm 17.0 \mathrm{~g} / \mathrm{L}$ (range 79-169 g/L) and 0.399 \pm 0.048 (range 0.239-0.509), respectively. The mean ratio of Lab-Hct to laboratory hemoglobin was $0.30 \pm 0.01$. A minority of subjects met criteria for anemia $(n=31,25 \%)$. Mean Lab-ECV was $27.1 \pm 4.7 \%$ (range 19.4-44.9\%).

POC analysis was successfully performed in 63\% $(n=77)$ of subjects on the first attempt, in $70 \%(n=86)$ within two attempts, and in $91 \%(n=112)$ within three attempts. The mean duration for POC analysis was $82 \pm 9$ s (range 70105 s). Mean POC-Hct was $0.395 \pm 0.044$ with no
Table 1 Baseline Characteristics

\begin{tabular}{ll}
\hline Characteristic & Subjects $(n=123)$ \\
\hline Age (years) & $47.7 \pm 14.1$ \\
Male & $52(42 \%)$ \\
BSA (m2) & $1.9 \pm 0.3$ \\
Heart rate (bpm) & $69 \pm 12$ \\
Indication for CMR & \\
Chemotoxicity & $34(28 \%)$ \\
Hypertrophic cardiomyopathy & $24(20 \%)$ \\
Anderson-Fabry disease & $11(9 \%)$ \\
Myocarditis/pericarditis & $10(8 \%)$ \\
Sarcoid & $8(7 \%)$ \\
Cardiomyopathy, cause unspecified & $17(14 \%)$ \\
Healthy subjects & $19(15 \%)$ \\
Field Strength & \\
1.5 T & $74(60 \%)$ \\
3 T & $49(40 \%)$
\end{tabular}

Data are mean \pm standard deviation or number of patients with percentage in parentheses

BPM beats per minute, $B S A$ body surface area, $C M R$ cardiovascular magnetic resonance

Table 2 Hemoglobin, Hematocrit and CMR Results

\begin{tabular}{|c|c|}
\hline Measurement & Subjects $(n=123)$ \\
\hline \multicolumn{2}{|l|}{ CMR Values } \\
\hline LVEDV (mL) & $152($ (QR 126-184) \\
\hline Indexed LVEDV $\left(\mathrm{mL} / \mathrm{m}^{2}\right)$ & 80 (IQR 71-96) \\
\hline LVESV (mL) & 64 (IQR 50-80) \\
\hline Indexed LVESV $\left(\mathrm{mL} / \mathrm{m}^{2}\right)$ & 34 (IQR 27-43) \\
\hline LVSV (mL) & $82(I Q R$ 69-103) \\
\hline LVEF (\%) & 58 (IQR 52-64) \\
\hline \multicolumn{2}{|l|}{ Laboratory Values } \\
\hline Laboratory hemoglobin (g/L) & $134.1 \pm 17.0$ \\
\hline Laboratory hematocrit & $0.399 \pm 0.048$ \\
\hline Laboratory ECV (\%) & $27.1 \pm 4.7$ \\
\hline \multicolumn{2}{|l|}{ POC Values } \\
\hline POC hemoglobin (g/L) & $131.6 \pm 14.7$ \\
\hline POC hematocrit & $0.395 \pm 0.044$ \\
\hline POC ECV (\%) & $27.3 \pm 4.8$ \\
\hline \multicolumn{2}{|l|}{ Synthetic Values } \\
\hline Synthetic hematocrit & $0.398 \pm 0.031$ \\
\hline Synthetic ECV (\%) & $26.5 \pm 4.3$ \\
\hline Elevated Laboratory ECV ( $\geq 30 \%$ ) & $31(25 \%)$ \\
\hline Anemia & $31(25 \%)$ \\
\hline
\end{tabular}

Data are mean \pm standard deviation, median and interquartile range (IQR), or number of patients with percentage in parentheses

$C M R$ cardiovascular magnetic resonance, ECV extracellular volume, $L V E D V$ left ventricular end diastolic volume, LVEF left ventricular ejection fraction, LVESV left ventricular end systolic volume, LVSV left ventricular stroke volume, $P O C$ point-of-care 
significant difference between POC-Hct and Lab-Hct $(p=0.149)$. Mean POC-ECV was $27.3 \pm 4.8 \%$ with no significant difference between POC-ECV and LabECV $(p=0.106)$. There was good correlation between laboratory and POC measures of hematocrit $(r=0.81$, $p<0.001)$ and excellent correlation between laboratory and POC measures of ECV $(r=0.94, p<0.001)$, Fig. 2a. On Bland-Altman analysis, there was minimal bias for POC-ECV in comparison to Lab-ECV with modest precision (bias $=-0.18 \%, 95 \% \mathrm{CI}[-2.85,2.49]$, confidence limit 5.34\%), Fig. 3a.

Synthetic-Hct was derived from blood pool T1 values in all subjects imaged at $1.5 \mathrm{~T}(n=74)$. There was no statistically significant difference between Lab-Hct and syntheticHct $(0.392 \pm 0.042$ vs. $0.398 \pm 0.031, p=0.074)$ or Lab-ECV and synthetic-ECV $(26.7 \pm 4.4 \%$ vs. $26.5 \pm 4.3 \%, p=0.084)$ in the subgroup imaged at $1.5 \mathrm{~T}$. There was moderate correlation between laboratory and synthetic measures of hematocrit $(r=0.60, p<0.001)$ and excellent correlation between laboratory and synthetic measures of ECV $(r=0.92$, $\mathrm{p}<0.001$ ), Fig. 2b. On Bland-Altman analysis, there was slightly larger bias for synthetic-ECV in comparison to LabECV and slightly wider limits of agreement (bias $=0.23 \%$, $95 \%$ CI [- 2.82, 3.27], confidence limit 6.09\%), Fig. 3b.

\section{Sensitivity analysis}

There was no statistically significant difference between POC-ECV and Lab-ECV when analysis was restricted to different subgroups, including subjects imaged at $1.5 \mathrm{~T}$ ( $n$ $=74, p=0.410)$, imaged at $3 \mathrm{~T}(n=49, p=0.120)$, females $(n=71, p=0.458)$, males $(n=52, p=0.087)$, healthy subjects $(n=19, p=0.376)$, and patients $(n=104, p=0.164)$. However, there was a significant difference between POCECV and Lab-ECV when analysis was restricted to subjects with anemia $(n=31, p=0.009)$.
There was no significant difference between syntheticECV and Lab-ECV when analysis was restricted to males $(n=22, p=0.249)$ and healthy subjects $(n=12, p=0.182)$. However, there were significant differences between synthetic-ECV and Lab-ECV when analysis was restricted to females $(n=52, p=0.008)$, patients $(n=62, p=0.018)$, and subjects with anemia $(n=22, p=0.001)$, suggesting that synthetic-ECV may be less robust compared to POCECV.

\section{Diagnostic performance}

ROC curves were used to evaluate the diagnostic performance of POC-ECV and synthetic-ECV in comparison to Lab-ECV as the reference standard using a cut-off of Lab-ECV $\geq 30 \%$ as abnormal. POC-ECV had good diagnostic test performance for discriminating Lab-ECV $\geq 30 \%$ (sensitivity $85 \%$, specificity $96 \%$, accuracy 94\%, and area under the curve (AUC) 0.902, $95 \% \mathrm{CI}[0.829,0.976])$. Synthetic-ECV had moderate diagnostic test performance (sensitivity $71 \%$, specificity $98 \%$, accuracy 93\%, and AUC 0.849, 95\% CI[0.725, 0.973]). There was no significant difference in AUC between the two noninvasive measures when analysis was restricted to studies performed at $1.5 \mathrm{~T}(p=0.629)$.

\section{Test-retest and inter-observer agreement}

Excellent test-retest (ICC 0.951, 95\%CI[0.905, 0.977]) and inter-observer agreement (ICC 0.900, 95\%CI[0.719, 0.965]) were demonstrated for POC-Hct. Excellent testretest (ICC 0.994, 95\%CI $[0.987,0.997]$ ) and interobserver agreement (ICC 0.974, 95\%CI[0.929, 0.991]) were demonstrated for POC-ECV.

\section{Comparison of POC devices}

In the group of subjects who underwent parallel hematocrit assessment with two POC devices and

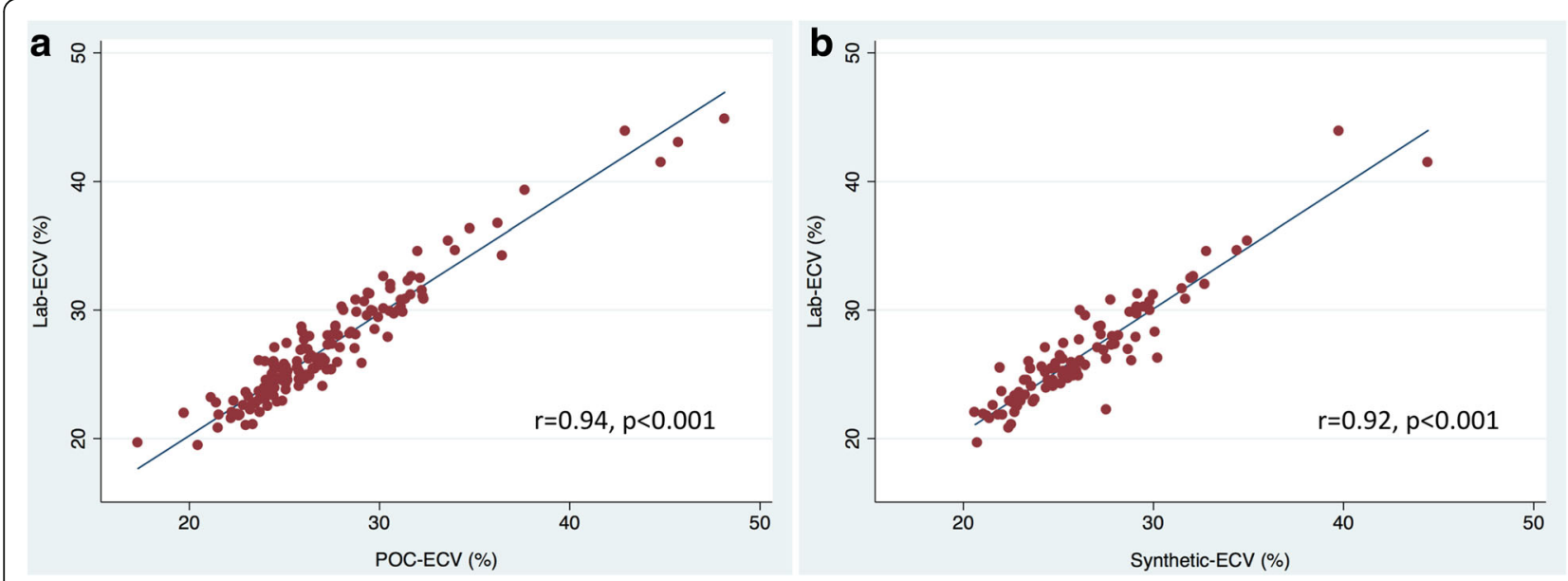

Fig. 2 Linear correlation between laboratory and point-of-care (POC) derived extracellular volume (ECV) (a) and laboratory and synthetic derived ECV (b) 

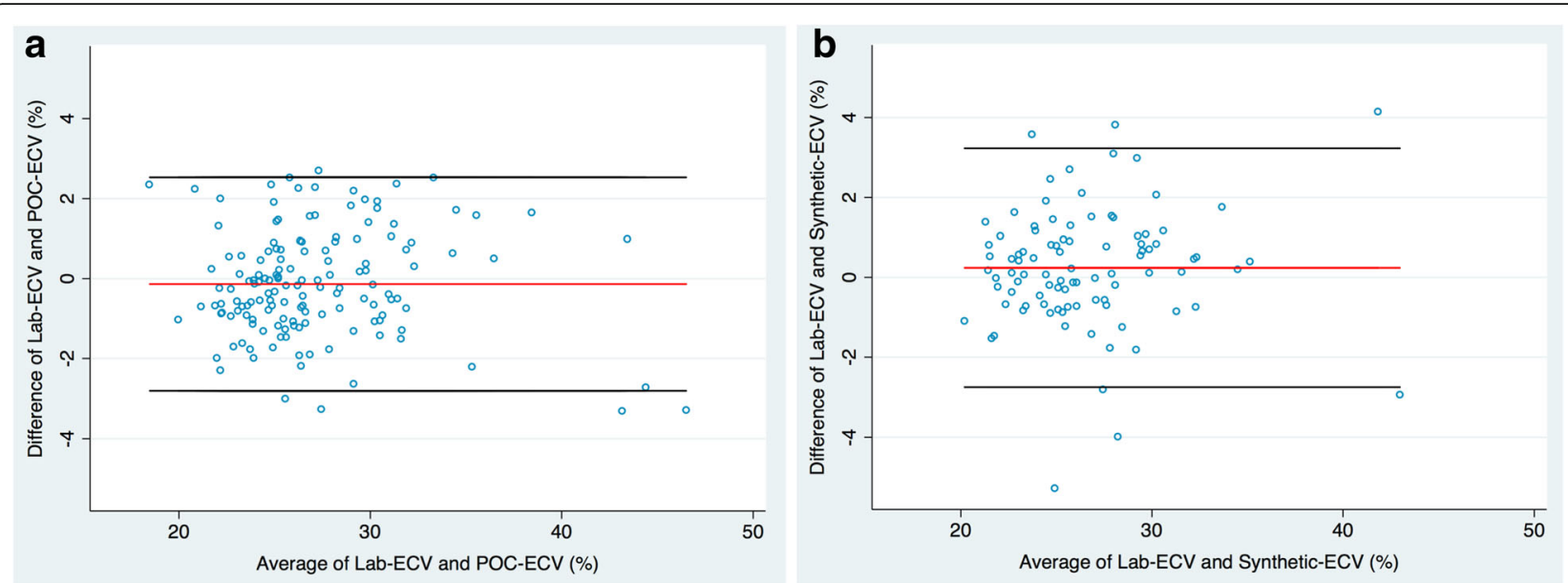

Fig. 3 Bland-Altman plots of the mean differences between laboratory and point-of-care (POC) derived extracellular volume (ECV) (a) and laboratory and synthetic derived ECV (b). For each parameter in (a) and (b), the average of measurements from both techniques is plotted on the $x$-axis and the difference between techniques is plotted on the $y$-axis. The solid red horizontal line plots the mean difference and the solid black lines indicated the limits of agreement (differences from the mean of 1.96 SDs) for each parameter

laboratory determination, there was no significant difference in hematocrit assessed using the Pronto device $(0.408 \pm 0.045)$ compared to the Pronto- $7^{\circ}$ device $(0.392 \pm 0.045, p=0.135)$ and compared to Lab-Hct $(0.405 \pm 0.044, p=0.830)$. Similarly, there was no significant difference in ECV derived using hematocrit from the Pronto ${ }^{\circ}$ POC device $(24.3 \pm 3.0 \%)$ compared to ECV derived using hematocrit from the Pronto- $7^{\circ}$ POC device $(25.0 \pm 2.9 \%, p=0.124)$ and compared to ECV derived from Lab-Hct $(24.3 \pm 2.2 \%, p=0.959)$.

\section{Discussion}

The results of this prospective study demonstrate that accurate and reproducible CMR ECV values can be calculated with input of hematocrit measured using a noninvasive POC device, potentially eliminating the need for blood collection by venipuncture for the purpose of ECV analysis. POC-ECV values correlate strongly with conventionally calculated ECV using laboratory hematocrit as the reference standard, with good diagnostic test performance, minimal bias and good agreement with Lab-ECV in both genders. Synthetic-ECV has slightly larger bias, wider limits of agreement and is less robust on sensitivity analyses when compared to POC-ECV.

There is growing interest in quantifying myocardial $\mathrm{ECV}$ as a marker of diffuse interstitial myocardial changes including fibrosis [22]. Myocardial ECV correlates with histologic measures of diffuse myocardial fibrosis [23, 24], and is independently associated with adverse outcomes including mortality [4]. However, clinical implementation of myocardial ECV may be limited by the requirement for timely hematocrit assessment [25]. Hematocrit is conventionally assessed by a peripheral blood sample obtained by venipuncture which is often inconvenient and uncomfortable for the patient. Several methods of noninvasive hematocrit evaluation have recently become commercially available which could be implemented to streamline CMR ECV evaluation.

Noninvasive POC devices provide rapid on the spot measures of hemoglobin without the need for blood sampling $[15,26]$. Most noninvasive POC devices rely on a spectrophotometer finger probe sensor to analyze light absorption characteristics of different hemoglobin species [27]. Prior studies utilizing earlier POC devices reported conflicting results [28, 29]. However, comparison of earlier results to current POC device performance is limited given ongoing software and hardware revisions [30]. More recent generations of POC devices (including the device used in this study), have been evaluated in subjects in a variety of clinical settings $[9,31,32]$ and in healthy volunteers $[33,34]$, with good correlation between POC hemoglobin and laboratory reference measures. A recent large meta-analysis evaluating the accuracy and precision of current POC devices against central laboratory hemoglobin measurements reported a pooled random-effects mean difference of POC minus central laboratory hemoglobin values of $0.10 \pm 1.37 \mathrm{~g} / \mathrm{dL}$ (limits of agreement -2.59 to $2.80 \mathrm{~g} / \mathrm{dL}$ ) [15]. Similarly, the results of our study demonstrate a small mean difference between POC-ECV and Lab-ECV of $-0.18 \%$ with relatively narrow limits of agreement ( -2.85 to $2.49 \%$ ). Notably, previous publications have not evaluated the diagnostic performance of POC derived ECV values, which is an important strength of the current study.

We evaluated POC device performance in the calculation of myocardial ECV in a relatively diverse cohort of 
subjects, and therefore results are applicable in a relatively broad range of clinical and research settings. POC analysis was quick, with most measurements acquired with a single analysis attempt. This noninvasive measure of hematocrit was easily incorporated into the CMR workflow and can be implemented in myocardial ECV calculation with the following caveats. POC analysis was not possible in a small subset of subjects, including subjects with applied gel nail polish, marked hand tremor and poor peripheral perfusion, which are recognized limitations of POC device analysis [28, 35]. Additionally, in the subset of subjects with anemia, there was a significant difference between laboratory and POC ECV values. This may be explained by the fact that low hemoglobin levels have been shown to impair perfusion analysis [36]. Given this finding, POC derived hematocrit is not recommended for CMR ECV calculation in subjects with anemia. This finding can be further explored in future studies that include a larger number of subjects with anemia.

A few recent studies have reported synthetic derivation of hematocrit and ECV based on the linear relationship between hematocrit and non-contrast R1 of blood [11, 13]. Automated inline derivation of synthetic hematocrit with subsequent calculation of myocardial ECV could potentially improve workflow, eliminating the need to acquire an invasive hematocrit value at the time of CMR. Treibel et al. demonstrated moderate correlation between synthetic and laboratory hematocrit $\left(\mathrm{r}^{2}=0.51-\right.$ $0.45, p<0.001$ ) and strong correlation between synthetic and laboratory derived ECV $\left(\mathrm{r}^{2}=0.97, p<0.001\right)$ [11]. Our study similarly demonstrates excellent correlation between synthetic and laboratory ECV, although the mean difference between synthetic-ECV and Lab-ECV was slightly greater and the limits of agreement were slightly wider when compared to POC-ECV. A recent study investigating synthetic ECV in a pediatric population reported 41 (25\%) false negatives and 4 (2\%) false positives for mid-septal synthetic ECV using a published model and cut-off value of $28.5 \%$ [13]. The authors conclude that use of synthetic hematocrit for the calculation of ECV results in miscategorization of individual patients. Similarly, the results of the current study demonstrate that synthetic-ECV values are less robust on sensitivity analysis compared to POC-ECV, with significant differences between laboratory and synthetic ECV values when restricted to females, patients and those with anemia. Synthetic hematocrit and ECV values are affected by factors that affect non-contrast blood pool $\mathrm{T} 1$ values, such as changes in oxygen and iron concentration and body temperature [37-40]. There are also important practical limitations to synthetic analysis, as formulas to calculate synthetic hematocrit should be derived at each institution in a large sample of patients imaged on each individual scanner and with each specific acquisition scheme to which the results will be applied [12]. We conclude that synthetic-ECV is less robust compared to POC-ECV, and requires further evaluation prior to clinical implementation.

Our study has a number of limitations. First, the noninvasive POC device used in this study became commercially unavailable after this study was completed. However, multiple other non-invasive hemoglobin monitoring devices are currently available, including Pronto Pulse CO-Oximeter (Masimo Personal Health), Radical$7^{\circ}$ Pulse CO-Oximeter (Masimo Personal Health), NBM-200 (OrSense, Petah-Tikva, Israel) and Haemospect (MRB Optical Systems GmbH \& Co. KG, Wuppertal, Germany). Several prior studies have compared hemoglobin results from the Pronto- $7^{\circ}$ device used in this study and NBM-200 device, with similar accuracy and bias reported between devices [26, 41-43]. A recent meta-analysis evaluating the accuracy of non-invasive hemoglobin monitoring devices reported that pooled mean differences and standard deviations were similar among three devices included in the analysis (Radical- $7^{\circ}$, Pronto- $7^{\circ}$, and NBM-200 devices) [15]. We demonstrate no significant difference in hematocrit or ECV assessed using the Pronto Pulse CO-Oximeter compared to the Pronto- $7^{\circ}$ Pulse CO-Oximeter and to laboratory values. These results suggest that currently available noninvasive hemoglobin monitoring devices will most likely lead to similar findings as reported in this study. Second, diurnal and monthly within-subject fluctuations in hematocrit are a recognized phenomenon, estimated at $3 \%[44,45]$, with additional variations in hematocrit values linked to season, age, hydration and physical activity [46-49]. Our study used a single laboratory hematocrit measure within $24 \mathrm{~h}$ of CMR as the reference standard. It is possible that slight fluctuations in hematocrit between the time of CMR, POC device acquisition and peripheral blood sampling for laboratory analysis could have influenced the results. The median interval between laboratory hematocrit analysis and CMR was only $1.3 \mathrm{~h}$ and therefore the impact of potential variations in hematocrit between analysis methods is likely minimal. Third, non-invasive hemoglobin measurements may vary between POC devices and settings. However, a recent meta-analysis has demonstrated good agreement in hemoglobin between various POC devices [15]. The presence of an arrhythmia, including atrial fibrillation, was not recorded at the time of CMR. Therefore, the accuracy of POC devices in the setting of an arrhythmia has not been evaluated. Finally, the sample size was modest and all data were acquired at a single institution. Further multi-center studies including a larger number of patients with anemia should be performed to validate these results. 


\section{Conclusions}

CMR ECV calculated with input of hematocrit measured using a noninvasive POC device is accurate and reproducible compared to conventionally calculated ECV using laboratory hematocrit, potentially overcoming an important barrier to clinical implementation of ECV measurements. Further evaluation of synthetic $\mathrm{ECV}$ is required prior to clinical implementation.

\section{Abbreviations}

AUC: Area under the curve; bSSFP: Balanced steady-state free precession; CMR: Cardiovascular magnetic resonance; ECV: Extracellular volume: Hct: Hematocrit; ICC: Intra-class correlation coefficient; IQR: Interquartile range; Lab-ECV: Laboratory extracellular volume; Lab-Hct: Laboratory hematocrit; LGE: Late gadolinium enhancement; LV: Left ventricle/left ventricular; MOLLI: MOdified Look-Locker Inversion; POC: Point-of-care; POCECV: Point-of-care extracellular volume; POC-Hct: Point-of-care hematocrit; ROC: Receiver operating curve; Synthetic-ECV: Synthetic extracellular volume; Synthetic-Hct: Synthetic hematocrit

\section{Acknowledgements}

Rosanna Chan, Research MRI Technologist.

\section{Funding}

KH: Radiological Society of North America Research Scholar Grant (RSCH1608) - salary support for corresponding author. $\mathrm{KH}$ : Academic Innovation Fund (AIF) grant from the Joint Department of Medical Imaging, University of Toronto - support for design of the study and data collection, data analysis, and POC device acquisition.

RW: Canadian Institutes of Health Research Operating Grant (MOP 119353) - salary support.

PT: Canadian Institutes of Health Research New Investigator Award (FRN

147814) - support for data collection and analysis.

\section{Availability of data and materials}

The datasets used and/or analysed during the current study are available from the corresponding author on reasonable request.

\section{Authors' contributions}

SR and $\mathrm{KH}$ were responsible for conception and design of the study. SR and GK contributed to subject recruitment and data acquisition. SR and $\mathrm{KH}$ analyzed and interpreted the data. SR, KH and PT contributed to statistical analysis. All authors made substantial contributions to analysis and interpretation of data. SR and $\mathrm{KH}$ drafted the manuscript. All authors critically revised and reviewed the manuscript. All authors read and approved the final manuscript and agree to be accountable for all aspects of the work.

\section{Ethics approval and consent to participate}

Institutional research and ethics board approval was obtained for this prospective study (University Health Network Research Ethics Board). Written informed consent was obtained from all study subjects.

\section{Consent for publication}

Not applicable.

\section{Competing interests}

The authors declare that they have no competing interests.

\section{Publisher's Note}

Springer Nature remains neutral with regard to jurisdictional claims in published maps and institutional affiliations.

\section{Author details}

'Department of Medical Imaging, Toronto General Hospital, University Health Network, University of Toronto, 585 University Ave, 1PMB-298, Toronto, ON M5G 2N2, Canada. ${ }^{2}$ Division of Cardiology, Department of Medicine, Peter Munk Cardiac Center, Toronto General Hospital, University of Toronto, Toronto, Canada.
Received: 12 September 2017 Accepted: 5 March 2018

Published online: 15 March 2018

\section{References}

1. de Meester de Ravenstein C, Bouzin C, Lazam S, Boulif J, Amzulescu M, Melchior J, Pasquet A, Vancraeynest D, Pouleur A-C, Vanoverschelde J-LJ, Gerber BL. Histological validation of measurement of diffuse interstitial myocardial fibrosis by myocardial extravascular volume fraction from modified look-locker imaging (MOLLI) T1 mapping at 3 T. J Cardiovasc Magn Reson. 2015;17:48.

2. Flett AS, Hayward MP, Ashworth MT, Hansen MS, Taylor AM, Elliott PM, McGregor C, Moon JC. Equilibrium contrast cardiovascular magnetic resonance for the measurement of diffuse myocardial fibrosis: preliminary validation in humans. Circulation. 2010;122:138-44.

3. Diao K-Y, Yang Z-G, Xu H-Y, Liu X, Zhang Q, Shi K, Jiang L, Xie L-J, Wen L-Y, Guo Y-K. Histologic validation of myocardial fibrosis measured by T1 mapping: a systematic review and meta-analysis. J Cardiovasc Magn Reson. 2016;18:1407.

4. Wong TC, Piehler K, Meier CG, Testa SM, Klock AM, Aneizi AA, Shakesprere J, Kellman P, Shroff SG, Schwartzman DS, Mulukutla SR, Simon MA, Schelbert EB. Association between extracellular matrix expansion quantified by cardiovascular magnetic resonance and short-term mortality. Circulation. 2012;126:1206-16.

5. Wong TC, Piehler KM, Kang IA, Kadakkal A, Kellman P, Schwartzman DS, Mulukutla SR, Simon MA, Shroff SG, Kuller LH, Schelbert EB. Myocardial extracellular volume fraction quantified by cardiovascular magnetic resonance is increased in diabetes and associated with mortality and incident heart failure admission. Eur Heart J. 2014;35:657-64.

6. Ambale-Venkatesh B, Lima JA, Cardiac MRI. A central prognostic tool in myocardial fibrosis. Nat Rev Cardiol. 2014;12:18-29.

7. Jellis $\mathrm{CL}$, Kwon DH. Myocardial T1 mapping: modalities and clinical applications. Cardiovasc Diagn Ther. 2014;4:126-37.

8. Berkow L, Rotolo S, Mirski E. Continuous noninvasive hemoglobin monitoring during complex spine surgery. Anesth Analg. 2011;113:1396-402.

9. Joseph B, Pandit V, Aziz H, Kulvatunyou N, Zangbar B, Tang A, O' keeffe T, Jehangir Q, Snyder K, Rhee P. Transforming hemoglobin measurement in trauma patients: noninvasive spot check hemoglobin. J Am Coll Surg. 2015; 220:93-8.

10. Frasca D, Dahyot-Fizelier C, Catherine K, Levrat Q, Debaene B, Mimoz O. Accuracy of a continuous noninvasive hemoglobin monitor in intensive care unit patients. Crit Care Med. 2011;39:2277-82

11. Treibel TA, Fontana M, Maestrini $V$, Castelletti S, Rosmini S, Simpson J, Nasis A, Bhuva AN, Bulluck H, Abdel-Gadir A, White SK, Manisty C, Spottiswoode BS, Wong TC, Piechnik SK, Kellman P, Robson MD, Schelbert EB, Moon JC. Automatic measurement of the myocardial interstitium: synthetic extracellular volume quantification without hematocrit sampling. J Am Coll Cardiol Img. 2016;9:54-63.

12. Bluemke DA, Kawel-Boehm N. Can a MR imaging scanner accurately measure hematocrit to determine ECV fraction? J Am Coll Cardiol Img. 2016:9:64-6.

13. Raucci FJ, Parra DA, Christensen JT, Hernandez LE, Markham LW, Xu M, Slaughter JC, Soslow JH. Synthetic hematocrit derived from the longitudinal relaxation of blood can lead to clinically significant errors in measurement of extracellular volume fraction in pediatric and young adult patients. J Cardiovasc Magn Reson. 2017:19:58

14. Messroghli DR, Greiser A, Fröhlich M, Dietz R, Schulz-Menger J. Optimization and validation of a fully-integrated pulse sequence for modified look-locker inversion-recovery (MOLLI) T1 mapping of the heart. J Magn Reson Imaging. 2007:26:1081-6.

15. Kim S-H, Lilot M, Murphy LS-L, Sidhu KS, Yu Z, Rinehart J, Cannesson M. Accuracy of continuous noninvasive hemoglobin monitoring. Anesth Analg. 2014;119:332-46.

16. Beutler $E$, Waalen J. The definition of anemia: what is the lower limit of normal of the blood hemoglobin concentration? Blood. 2006;107:1747-50.

17. Anaemias N. Report of a WHO scientific group. World health organ tech. Ser. 1968:405:5-37.

18. Arheden H, Saeed M, Higgins CB, Gao DW, Bremerich J, Wyttenbach R, Dae MW, Wendland MF. Measurement of the distribution volume of gadopentetate dimeglumine at echo-planar MR imaging to quantify myocardial infarction: comparison with 99mTc-DTPA autoradiography in rats. Radiology. 1999;211:698-708. 
19. Neilan TG, Coelho-Filho OR, Shah RV, Abbasi SA, Heydari B, Watanabe E, Chen Y, Mandry D, Pierre-Mongeon F, Blankstein R, Kwong RY, JeroschHerold M. Myocardial extracellular volume fraction from T1 measurements in healthy volunteers and mice. J Am Coll Cardiol Img. 2013;6:672-83.

20. Liu S, Han J, Nacif MS, Jones J, Kawel N. Diffuse myocardial fibrosis evaluation using cardiac magnetic resonance T1 mapping: sample size considerations for clinical trials. J Cardiovasc Magn. 2012;14:90.

21. Kellman P, Wilson JR, Xue H, Bandettini WP, Shanbhag SM, Druey KM, Ugander M, Arai AE. Extracellular volume fraction mapping in the myocardium, part 2: initial clinical experience. J Cardiovasc Magn Reson. 2012;14:64.

22. Taylor AJ, Salerno M, Dharmakumar R, Jerosch-Herold M. T1 mapping: basic techniques and clinical applications. J Am Coll Cardiol Img. 2016;9:67-81.

23. Miller CA, Naish JH, Bishop P, Coutts G, Clark D, Zhao S, Ray SG, Yonan N, Williams SG, Flett AS, Moon JC, Greiser A, Parker GJM, Schmitt M. Comprehensive validation of cardiovascular magnetic resonance techniques for the assessment of myocardial extracellular volume. Circ Cardiovasc Imaging. 2013;6:373-83.

24. Siepen FAD, Buss SJ, Messroghli D, Andre F, Lossnitzer D, Seitz S, Keller M, Schnabel PA, Giannitsis E, Korosoglou G, Katus HA, Steen H. T1 mapping in dilated cardiomyopathy with cardiac magnetic resonance: quantification of diffuse myocardial fibrosis and comparison with endomyocardial biopsy. Eur Heart J Cardiovasc Imaging. 2015;16:210-6.

25. Moon JC, Messroghli DR, Kellman P, Piechnik SK, Robson MD, Ugander M, Gatehouse PD, Arai AE, Friedrich MG, Neubauer S, Schulz-Menger J, Schelbert EB. Society for Cardiovascular Magnetic Resonance Imaging, cardiovascular magnetic resonance working Group of the European Society of cardiology. Myocardial T1 mapping and extracellular volume quantification: a Society for Cardiovascular Magnetic Resonance (SCMR) and CMR working Group of the European Society of cardiology consensus statement. J Cardiovasc Magn Reson. 2013;15:92.

26. Gayat E, Aulagnier J, Matthieu E, Boisson M, Fischler M. Non-invasive measurement of hemoglobin: assessment of two different point-of-care technologies. PLoS One. 2012;7:e30065.

27. McMurdy JW, Jay GD, Suner S, Crawford G. Noninvasive optical, electrical, and acoustic methods of total hemoglobin determination. Clin Chem. 2008; 54:264-72.

28. Miller RD, Ward TA, Shiboski SC, Cohen NHA. Comparison of three methods of hemoglobin monitoring in patients undergoing spine surgery. Anesth Analg. 2011;112:858-63.

29. Riess ML, Pagel PS. Noninvasively measured hemoglobin concentration reflects arterial hemoglobin concentration before but not after cardiopulmonary bypass in patients undergoing coronary artery or valve surgery. J Cardiothorac Vasc Anesth. 2016;30:1167-71.

30. Hiscock R. Systematic review and meta-analysis of method comparison studies of Masimo pulse co-oximeters (Radical- $7^{\mathrm{TM}}$ or Pronto- $7^{\mathrm{TM}}$ ) and HemoCue $^{\circledast}$ absorption spectrometers (B-hemoglobin or 201+) with laboratory haemoglobin estimation. Anaesth Intensive Care. 2015;43:3.

31. DeBarros M, Shawhan R, Bingham J, Sokol K, Izenberg S, Martin M. Assessing serum hemoglobin levels without venipuncture: accuracy and reliability of Pronto-7 noninvasive spot-check device. Amer J Surg. 2015;209:848-55.

32. Erdogan Kayhan G, Colak YZ, Sanli M, Ucar M, Toprak H. Accuracy of noninvasive hemoglobin monitoring by pulse co-oximeter during liver transplantation. Minerva Anestesiol. 2017;83(5):485-92.

33. Shah N, Osea EA, Martinez GJ. Accuracy of noninvasive hemoglobin and invasive point-of-care hemoglobin testing compared with a laboratory analyzer. Int J Lab Hematol. 2013;36:56-61.

34. Vyas KJ, Danz D, Gilman RH, Wise RA, León-Velarde F, Miranda JJ, Checkley W. Noninvasive assessment of excessive Erythrocytosis as a screening method for Chronic Mountain sickness at high altitude. High Alt Med Biol. 2015;16:162-8.

35. Yamaura K, Nanishi N, Higashi M, Hoka S. Effects of thermoregulatory vasoconstriction on pulse hemoglobin measurements using a co-oximeter in patients undergoing surgery. J Clin Anesth. 2014;26:643-7.

36. Khalafallah AA, Chilvers CR, Thomas M, Chilvers CM, Sexton M, Vialle M, Robertson IK. Usefulness of non-invasive spectrophotometric haemoglobin estimation for detecting low haemoglobin levels when compared with a standard laboratory assay for preoperative assessment. Br J Anaesth. 2015; 114:669-76.
37. Barth M, Moser E. Proton NMR Relaxation times of human blood samples at $1.5 \mathrm{~T}$ and implications for functional MRI. Cell Mol Biol (Noisy-le-grand). 1997:43:783-91.

38. Tadamura E, Hatabu H, Li W, Prasad PV, Edelman RR. Effect of oxygen inhalation on relaxation times in various tissues. J Magn Reson Imaging. 1997:7:220-5.

39. Yilmaz A, Bucciolini M, Longo G, Franciolini F, Ciraolo L, Renzi R. Determination of dependence of spin-lattice relaxation rate in serum upon concentration of added iron by magnetic resonance imaging. Clin Phys Physiol Meas. 1990;11:343-9.

40. Silvennoinen MJ, Kettunen MI, Kauppinen RA. Effects of hematocrit and oxygen saturation level on blood spin-lattice relaxation. Magn Reson Med. 2003:49:568-71.

41. Belardinelli A, Benni M, Tazzari PL, Pagliaro P. Noninvasive methods for haemoglobin screening in prospective blood donors. Vox Sang 16 ed. 2013; 105:116-20.

42. Ardin S, Störmer M, Radojska S, Oustianskaia L, Hahn M, Gathof BS. Comparison of three noninvasive methods for hemoglobin screening of blood donors. Transfusion. 2014;55:379-87.

43. Pajares-Herraiz AL, Rodriguez-Gambarte JD, Eguia-Lopez B, FernandezMaqueda CT. Coello de Portugal C, Flores-Sanz MDV. A comparative study of three non-invasive Systems for Measurement of hemoglobin with HemoCue system having coulter LH750 as reference value. Hematol Transfus Int J MedCrave Online. 2015;1:1-10.

44. Jones AR, Twedt D, Swaim W, Gottfried E. Diurnal change of blood count analytes in normal subjects. Am J Clin Pathol. 1996;106:723-7.

45. Pocock SJ, Ashby D, Shaper AG, Walker M, Broughton PM. Diurnal variations in serum biochemical and haematological measurements. J Clin Pathol. 1989;42:172-9

46. Wennesland R, Brown E, Hopper J, Hodges JL, Guttentag OE, Scott KG, Tucker IN, Bradley B. Red cell, plasma and blood volume in healthy men measured by radiochromium ( $\mathrm{Cr} 51)$ cell tagging and hematocrit: influence of age, somatotype and habits of physical activity on the variance after regression of volumes to height and weight combined. J Clin Invest. 1959; 38:1065-77.

47. Schumacher YO, Grathwohl D, Barturen JM, Wollenweber M, Heinrich L, Schmid A, Huber G, Haemoglobin KJ. Haematocrit and red blood cell indices in elite cyclists. Are the control values for blood testing valid? Int J Sports Med. 2000;21:380-5.

48. Thirup P. Haematocrit: within-subject and seasonal variation. Sports Med. 2003;33:231-43.

49. Schmidt W, Biermann B, Winchenbach P, Lison S, Böning D. How valid is the determination of hematocrit values to detect blood manipulations? Int J Sports Med. 2000;21:133-8.

\section{Submit your next manuscript to BioMed Central and we will help you at every step:}

- We accept pre-submission inquiries

- Our selector tool helps you to find the most relevant journal

- We provide round the clock customer support

- Convenient online submission

- Thorough peer review

- Inclusion in PubMed and all major indexing services

- Maximum visibility for your research

Submit your manuscript at www.biomedcentral.com/submit
Biomed Central 